(1)

CrossMark

\title{
Availability, price and affordability of anti-tuberculosis drugs in Europe: a TBNET survey
}

\author{
Gunar Günther ${ }^{1,2,5}$, Gabriela B. Gomez ${ }^{3,4,5}$, Christoph Lange ${ }^{1,2}$, Stephan Rupert ${ }^{3}$ \\ and Frank van Leth ${ }^{3}$ on behalf of the TBNET ${ }^{6}$
}

\begin{abstract}
Affiliations: ${ }^{1}$ Division of Clinical Infectious Diseases, German Center for Infection Research (DZIF) Clinical Tuberculosis Unit, Research Center, Borstel, Germany. ${ }^{2}$ Department of Medicine, University of Namibia School of Medicine, Windhoek, Namibia. ${ }^{3}$ Department of Global Health, Academic Medical Center, University of Amsterdam, Amsterdam Institute for Global Health and Development, Amsterdam, The Netherlands. ${ }^{4}$ Department of Global Health and Development, London School of Hygiene and Tropical Medicine, London, UK. ${ }^{5}$ Both authors contributed equally to the manuscript. ${ }^{6}$ For a list of TBNET contributors, see Acknowledgements section.
\end{abstract}

Correspondence: Frank van Leth, Amsterdam Institute for Global Health and Development, Trinity Building C Pietersbergweg 17, 1105 BM Amsterdam, The Netherlands. E-mail: f.vanleth@aighd.org

ABSTRACT Data on availability and cost of anti-tuberculosis (TB) drugs in relation to affordability at national level are scarce.

We performed a cross-sectional study on availability and cost of anti-TB drugs at major TB-reference centres in 37 European countries. Costs of standardised treatment regimens used for pan-sensitive TB, multidrug-resistant (MDR) TB, pre-extensively drug-resistant (XDR) TB, and XDR-TB were compared using a purchasing power analysis. Affordability was evaluated in relation to monthly national gross domestic products per capita (GDP).

At least one second-line injectable and either moxifloxacin or levofloxacin were available in all countries. Linezolid and clofazimine were available in $79 \%$ and $46 \%$ of the countries, respectively. Drug cost for XDR-TB was three-times more expensive than those for MDR-TB. The average price of treatment for pan-sensitive TB represented a maximum of $8.5 \%$ of the monthly GDP across countries, while for standard MDR-TB treatment this was $<30 \%$ in only six countries and more than $100 \%$ in four countries. Treatment of XDR-TB represented more than $100 \%$ of a month's GDP in all countries where the regimen was available.

High cost and limited availability of drugs for treatment of drug-resistant TB, particularly beyond resistance to first-line drugs, are a major impediment to successful TB control in Europe.

@ERSpublications

Limited availability and high cost of drugs hamper the treatment of drug-resistant tuberculosis in Europe http://ow.ly/CrhbM

Earn CME accreditation by answering questions about this article. You will find these at erj.ersjournals.com/misc/ cmeinfo.xhtml

Received: July 082014 | Accepted after revision: Sept 292014 | First published online: Nov 132014

Support statement: This work was supported by the European Commission's Seventh Framework Programme (FP7/ 2007-2013) under grant agreement FP7-223681, and the German Center for Infection Research, DZIF. The funders had no role in the design, implementation or analysis of the study, nor in the preparation or decision to publish the manuscript.

Conflict of interest: Disclosures can be found alongside the online version of this article at erj.ersjournals.com

Copyright @ERS 2015 


\section{Introduction}

There is little empirical evidence on the costs of tuberculosis (TB) treatment globally and in Europe in particular. Only recently, a systematic review assessed the cost of TB treatment in Europe to build a case for investment for the development of a new TB vaccine [1]. This review did not present a detailed description of the cost of drugs, which might differ considerably between countries due to differences in healthcare and setting-specific funding mechanisms. Whether the costs of drugs are perceived as affordable will depend for a large part on who is bearing the burden: the patient or the healthcare system. For the patient, out-of-pocket expenses and indirect costs are a strong driver of healthcare-seeking behaviour and adherence to treatment [2-4]; whereas, for the healthcare system, drug and service delivery costs are important considerations. The recent economic recession has shown that financial constraints on healthcare systems can have detrimental effects on the incidence of infectious disease $[5,6]$.

To gain information about the costs of TB medication in Europe, we collected data on drug costs at TB treatment centres, and assessed the availability and affordability of treatment regimens across almost all European countries.

\section{Methods}

TBNET is a network to promote clinically oriented research in the field of TB in Europe, through collaborative research [7]. Physicians in the network collected data during February-March 2013 through hospital pharmacies, dispensaries or, where applicable, through national TB programmes.

Prices were translated to the cost of a day's treatment with the recommended dosage for each drug, as required in the treatment of a person weighing $70 \mathrm{~kg}$ following the guidelines of the World Health Organization (WHO) and international consensus [8-10]. If prices of separate drugs were not available, we divided the cost of the fixed-dose combination (FDC) equally over the drugs that were part of the FDC.

Prices were converted to international dollars (I\$) using the purchasing power parity conversion factor (2012) in the World Bank's International Comparison Program database 11, facilitating direct comparison between countries and regimens. An I\$ would buy in a specific country the same amount of goods and services as a US\$ would buy in the USA. Prices in Euro are reported for reference only.

Availability was reported as the number and percentage of countries in which individual drugs were available during the data collection period. We defined preferred and alternative treatment regimens for drug-sensitive TB, multidrug-resistant (MDR)-TB (resistant to isoniazid and rifampicin), pre-extensively drug-resistant (XDR)-TB (resistant to at least one fluoroquinolone or a second-line injectable in addition to isoniazid and rifampicin), and XDR-TB (resistant to at least one fluoroquinolone, second-line injectable, isoniazid and rifampicin) following international guidelines (table 1).

TABLE 1 Preferred and alternative treatment regimens by resistance pattern

\begin{tabular}{|c|c|c|c|c|}
\hline \multicolumn{2}{|c|}{ Drug resistance } & Preference & Duration (months) & Regimen \\
\hline \multirow[t]{2}{*}{ Sensitive } & None & Preferred & 2 & HRZE \\
\hline & & & 4 & $\mathrm{HR}$ \\
\hline \multirow[t]{3}{*}{ MDR } & Isoniazid and rifampicin & Preferred & 8 & SL-inj, FQ, Pto/Eto, Cs/Trd, E, Z \\
\hline & & & 12 & FQ, Pto/Eto, Cs/Trd, E, Z \\
\hline & & Alternative & 8 & SL-inj, FQ, PAS, Cs/Trd, E, Z \\
\hline Pre-XDRinj & & Alternative & 20 & FQ, PAS, Cs/Trd, Lzd, E, Z \\
\hline \multirow[t]{4}{*}{ Pre-XDRfq } & MDR + resistance against FQs & Preferred & 8 & SL-inj, Pto/Eto, Cs/Trd, Lzd, Cfz, E, Z \\
\hline & & & 12 & Pto/Eto, Cs/Trd, Lzd, Cfz, E, Z \\
\hline & & Alternative & 8 & SL-inj, PAS, Cs/Trd, Lzd, Cfz, E, Z \\
\hline & & & 12 & PAS, Cs/Trd, Lzd, Cfz, E, Z \\
\hline XDR & MDR + resistance against SL-inj + FQs & Preferred & 20 & Pto/Eto, Cs/Trd, Lzd, Cfz, Amx-Clav, Mero, E, Z \\
\hline
\end{tabular}

MDR: multidrug-resistant; pre-XDRinj: MDR tuberculosis with additional resistance to second-line injectable; pre-XDRfq: MDR tuberculosis with aditional resistance to fluoroquinolone; H: isoniazid; R: rifampicin; Z: pyrazinamide; E: ethambutol; SL-inj: second-line injectable; FQ: fluoroquinolone; Pto/Eto: prothionamide/ethionamide; Cs/Trd: cycloserine/terizidone; PAS: para-aminosalicylic acid; Lzd: linezolid; Cfz: clofazimine; Amx-Clav: amoxicillin-clavulanic acid; Mero: meropenem. 
Affordability was reported as the percentage of the gross domestic product (GDP) per capita in I\$ required to purchase all drugs in the standardised treatment regimen [12]. This indicator was chosen to provide a reference point (GDP) when assessing how expensive a drug regimen is and comparing it across countries, with the implication that if it is very expensive, patients/healthcare systems might not be able or willing to afford it. Given the duration of treatment for MDR-TB and (pre-)XDR-TB extending to over one year, we assessed affordability for a month's treatment. For this we divided the total drug cost of each regime by its duration (20 months for MDR- and (pre-XDR)-TB regimens, 6 months for pan-sensitive TB regimens), and the GDP by 12 , after which we took the ratio of these two estimates to derive the percentage of a month's GDP per capita represented by a month of treatment. Analysis was conducted using STATA 12 (Stata Corp. College Station, TX, USA). Given the nature and approach of the data collected, formal ethical approval was not required for the study.

\section{Results}

All of the current 28 member states of the European Union were included in the analysis, except for Malta. We also included Serbia, Former Yugoslavic Republic of Macedonia (FYROM) and Montenegro (candidate member states); as well as Norway, Switzerland, Republic of Moldova, Ukraine, Bosnia and Herzegovina, Albania and Belarus. The data from Kosovo were excluded because all drugs were reported to be free-of-charge at the point of use.

\section{Availability}

The availability of drugs and regimens surveyed are depicted in figure 1.

All first-line drugs (isoniazid $(\mathrm{H})$, rifampicin $(\mathrm{R})$, pyrazinamide $(\mathrm{Z})$, ethambutol $(\mathrm{E})$ ) were available in all countries surveyed. 17 of the 37 countries indicated the use of FDC in first-line treatment. For only two countries, we needed to use the cost of FDCs to estimate the cost of individual drugs.

Amikacin was available in all counties except Bulgaria. Capreomycin was available in 22 (59\%) countries, while kanamycin was available in only nine $(24 \%)$ countries. Moxifloxacin and levofloxacin were available in $34(92 \%)$ and $32(86 \%)$ countries, respectively. All countries had at least one second-line injectable (SL-inj) and one fluoroquinolone (FQ) available.

Prothionamide/ethionamide (Pto/Eto) and cycloserine/terizidon (Cs/Trd) are essential in the preferred treatment regimen for MDR-TB, while para-aminosalicylic acid (PAS) is essential in the alternative MDR-TB regimen. These drugs were available in 31 (86\%), 32 (86\%) and 26 (70\%) countries, respectively. Three countries did not have any of these drugs available (Albania, Serbia, Greece), while another two countries (Bosnia and Herzegovina, Slovakia) had just one of these drugs available. A preferred MDR-TB regimen $(\mathrm{SL}-\mathrm{inj}+\mathrm{FQ}+\mathrm{Pto} / \mathrm{Eto}+\mathrm{Cs} / \mathrm{Trd})$, was available in $30(81 \%)$ countries. In only one of the remaining

FIGURE 1 Availability of selected drugs and treatment regimen. HRZE: isoniazid, rifampicin, pyrazinamide, ethambutol; SL-inj: second-line injection; $\mathrm{FQ}$ : fluoroquinolone; $\mathrm{PAS}$ : para-aminosalicylic acid; MDR: multidrug resistant; XDR: extensively drug resistant.

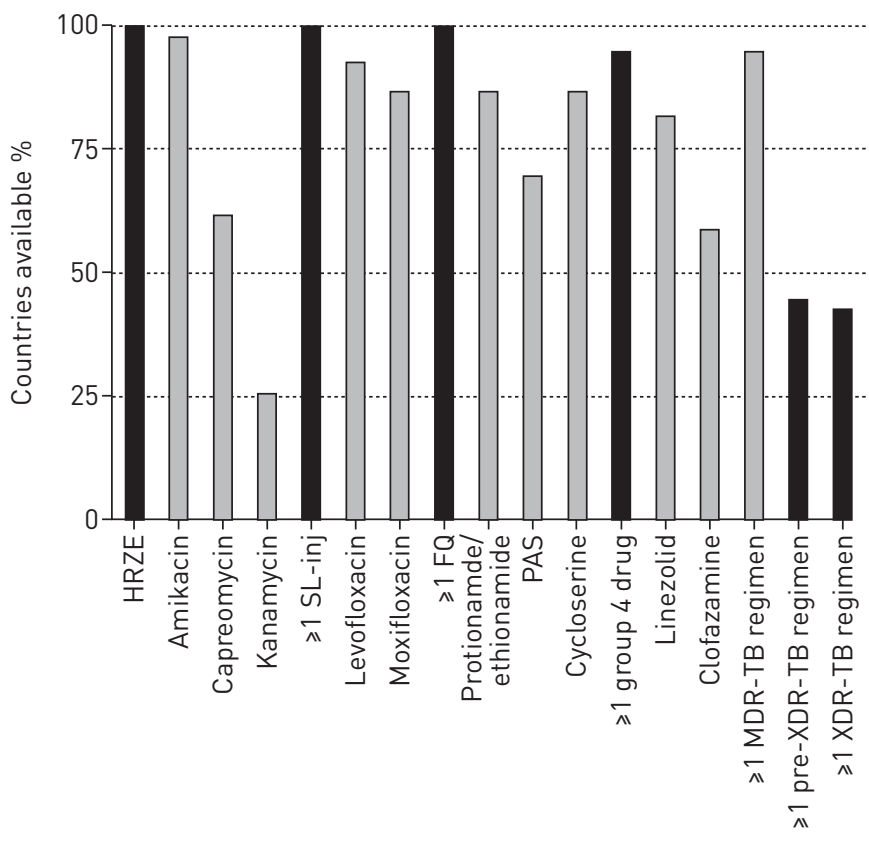


seven countries (Luxembourg), an alternative MDR-TB regimen in which PAS substitutes Pto/Eto was available, leaving six (16\%) countries without an adequate MDR-TB regimen.

The diagnosis of pre-XDR-TB is based on either resistance to SL-inj (pre-XDRinj) or FQs (pre-XDRfq) in addition to MDR-TB. A pre-XDRinj regimen contains linezolid (Lzd), while a pre-XDRfq regimen contains Lzd and clofazimine (Cfz). Lzd was available in 29 (78\%) countries, and Cfz in 17 (46\%). Five (14\%) countries had neither of these two drugs available. A preferred pre-XDRinj regimen (FQ+Pto/Eto+Cs/Trd $+\mathrm{Lzd})$ was available in $24(65 \%)$ of the countries. In just one of the 13 remaining countries (Luxembourg), an alternative pre-XDRinj regimen $(\mathrm{FQ}+\mathrm{PAS}+\mathrm{Cs} / \mathrm{Trd}+\mathrm{Lzd})$ was available. The preferred pre-XDRfq regimen (SL-inj+Pto/Eto+Cs/Trd+Lzd+Cfz) was available in just $16(43 \%)$, countries, while none of the countries without such a regimen had the alternative regimen (PAS instead of Pto/Eto).

Treatment of XDR-TB requires the use of preferably meropenem (Mero) and clavulanic acid, the latter available as amoxicillin-clavulanic acid (Amx-Clav) in Europe [10]. These drugs were available in 28 $(76 \%)$ and $31(84 \%)$ the countries surveyed, respectively. However a preferred XDR-TB regimen (Pto/Eto $+\mathrm{Cs} / \operatorname{Trd}+\mathrm{Lzd}+\mathrm{Cfz}+$ Amx-Clav+Mero) was available in just 13 (35\%) countries, while none of the countries without this regimen had the alternative regimen that substitutes PAS for Pto/Eto available.

Cost

The average prices of all individual drugs surveyed are reported in table 2 and those for treatment regimens in table 3.

Prices for first-line drugs were generally higher in North-West European countries than in Eastern or Southern European countries. The cost of a 6-month treatment regimen of HRZE for pan-sensitive TB ranged from 94 I\$ in the Republic of Moldova to 1165 I\$ in Switzerland.

Capreomycin (daily dose 67 I\$) was, on average, three-times as expensive as amikacin (daily dose 22 I\$), while the cost of a daily dose of kanamycin was on average just 6 I\$. There was no major price difference for a daily dose of levofloxacin or moxifloxacin, both costing approximately 7 I\$. Pto/Eto was on average one-third of the price of a daily dose of PAS (40 versus 103 I\$).

The price of a full 20-months MDR-TB regimen based on Pto/Eto and Cs/Trd was on average $32000 \mathrm{I \$}$, but could be as low as 4600 I\$ in the Republic of Moldova, and as high as 79300 I\$ in Finland, depending on the availability of the SL-inj and the FQs. The alternative regimen in which PAS is substituted for Pto/Eto was on average 14\% more expensive.

The preferred pre-XDR-TB regimens were, on average, almost twice as expensive as the preferred MDR-TB regimen, but this difference could be much larger. In Hungary, the preferred pre-XDRinj regimen was $182000 \mathrm{I}$ and the preferred pre-XDRfq regimen $207000 \mathrm{I}$, compared with $56000 \mathrm{I} \$$ for

TABLE 2 Average price per day treatment for individual drugs

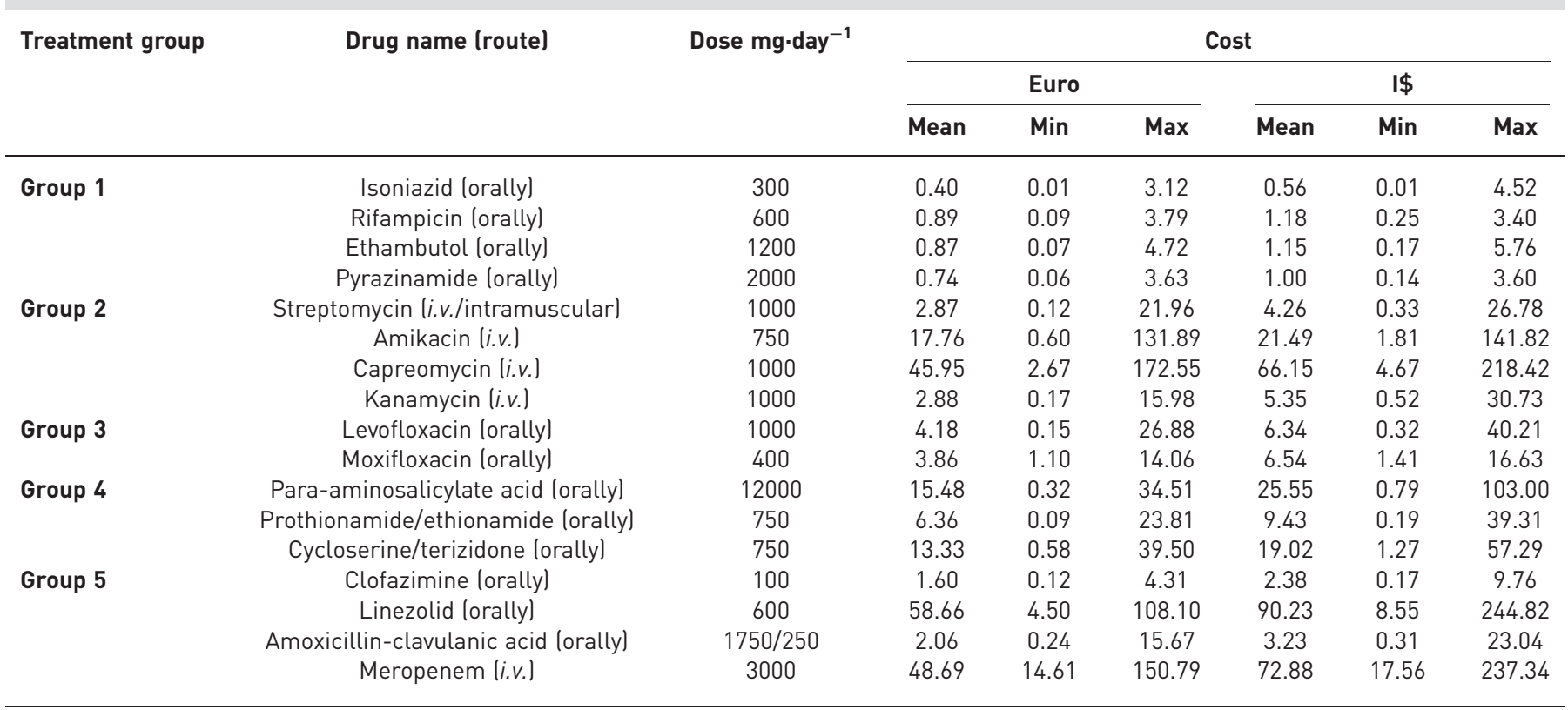


TABLE 3 Average drug costs per full treatment regimen where available

\begin{tabular}{|c|c|c|c|c|c|c|c|c|}
\hline \multirow[t]{3}{*}{ Resistance } & \multirow[t]{3}{*}{ Regimen } & \multirow[t]{3}{*}{ Countries $\mathrm{n}$} & \multicolumn{6}{|c|}{ Cost } \\
\hline & & & \multicolumn{3}{|c|}{ Euro } & \multicolumn{3}{|c|}{ I\$ } \\
\hline & & & Mean & Min & Max & Mean & Min & Max \\
\hline Pan-sensitive & Preferred & 37 & 334 & 41 & 1299 & 448 & 93 & 1164 \\
\hline MDR & Preferred & 30 & 23272 & 2112 & 77354 & 31715 & 4565 & 79247 \\
\hline MDR & Alternative & 23 & 23398 & 3136 & 72618 & 34656 & 6781 & 78083 \\
\hline \multirow[t]{2}{*}{ Pre-XDRfq } & Preferred & 16 & 67346 & 35507 & 106730 & 88199 & 61261 & 207152 \\
\hline & Alternative & 11 & 71506 & 29734 & 104023 & 95275 & 56498 & 213930 \\
\hline \multirow[t]{2}{*}{ XDR } & Preferred & 14 & 93962 & 56028 & 180237 & 123936 & 75587 & 329104 \\
\hline & Alternative & 9 & 90657 & 65031 & 148312 & 124887 & 82317 & 335882 \\
\hline
\end{tabular}

MDR: multidrug resistant; pre-XDRinj: MDR tuberculosis with additional resistance to second-line injectable; pre-XDRfq: MDR tuberculosis with aditional resistance to fluoroquinolone.

the preferred MDR-TB regimen. This is due to the very high price of Lzd in this country. In turn, the alternative pre-XDR-TB regimens were slightly more expensive than the preferred pre-XDR-TB regimens, with an increase of $9 \%$ and $8 \%$ for pre-XDRinj and pre-XDRfq, respectively.

The price of the 20-month preferred XDR-TB regimen (Pto/Eto+Cs/Trd+Lzd+Cfz+Amx-Clav+Mero) was at the lower end of the spectrum between 75000 and 90000 I\$ (Finland, France, Germany, Italy, the Netherlands and Sweden). Compared with these prices, the price in Hungary (329 000 I\$), where both Lzd and Mero are markedly more expensive than in other countries, is a clear outlier. The average price of the alternative XDR-TB regimen was 7\% higher than the preferred XDR-TB regimen.

\section{Affordability}

In all countries, the cost of a month's first-line regimen remained below 8.5\% of a month's GDP in 2012 . In seven countries, the cost was more than $5 \%$ of a month's GDP per capita, of which five were in Eastern or Southern Europe (Republic of Moldova (5.5\%), Slovenia (6.3\%), Bosnia and Herzegovina (6.7\%), Spain (6.8\%), Albania (8.3\%)) and two were in Northern or Western Europe (Finland (5.7\%), UK (6.0\%)). In Italy and Luxemburg, the cost of a month's course first-line regimen was below $1 \%$ of a month's GDP per capita but for different reasons: in Italy, the price of the first-line regimen was relatively inexpensive, while Luxemburg has the highest GDP of the countries surveyed.

The percentage of monthly GDP per capita represented by a month's treatment with the preferred MDR-TB regimen remained below 30\% in just three countries, while for 13 countries it was above $60 \%$, of which for seven countries above 100\% (Ukraine (101\%) Slovenia (114\%) Finland (124\%) Poland (146\%) Czech Republic (147\%) Hungary (155\%) Montenegro (182\%)).

The alternative MDR-TB regimen based on PAS instead of Pto/Eto represented up to 60\% of a month's GDP per capita in most countries. In eight countries, this percentage was above $100 \%$ (Ukraine (103\%), Republic of Moldova (119\%), Finland (122\%), Slovenia (133\%), Czech Republic (137\%), Hungary (162\%), FYROM (208\%), Montenegro (268\%)).

The percentage of a month's GDP represented by pre-XDR-TB regimens was high for both preferred and alternative regimens, as well as for both pre-XDRinj and pre-XDRfq regimens. In all four scenarios, the percentage could be as high as $400 \%$. For the pre-XDRinj regimens, the percentage was below $100 \%$ in just seven and four countries for the preferred and alternative regimens, respectively. This was even less frequent for pre-XDRfq regimens, where the percentage of a month's GDP represented by the regimen was below $100 \%$ in five and zero countries for the preferred and alternative regimens, respectively.

A month's treatment with the preferred 20-month XDR-TB regimen represented more than $100 \%$ of a month's GDP per capita in all countries where available $(n=14)$. The percentage ranged from $104 \%$ in Norway to $915 \%$ in Hungary. For the alternative regimen in which PAS substitutes Pto/Eto, the represented monthly GDP per capita ranged from $122 \%$ in Germany to $934 \%$ in Hungary among the nine countries where such regimen was available.

Figure 2 depicts the availability and affordability of each of the regimens for each of the countries surveyed. Countries are grouped by annual GDP per capita. It shows that regimens for the treatment of 


\section{Annual GDP per capital}

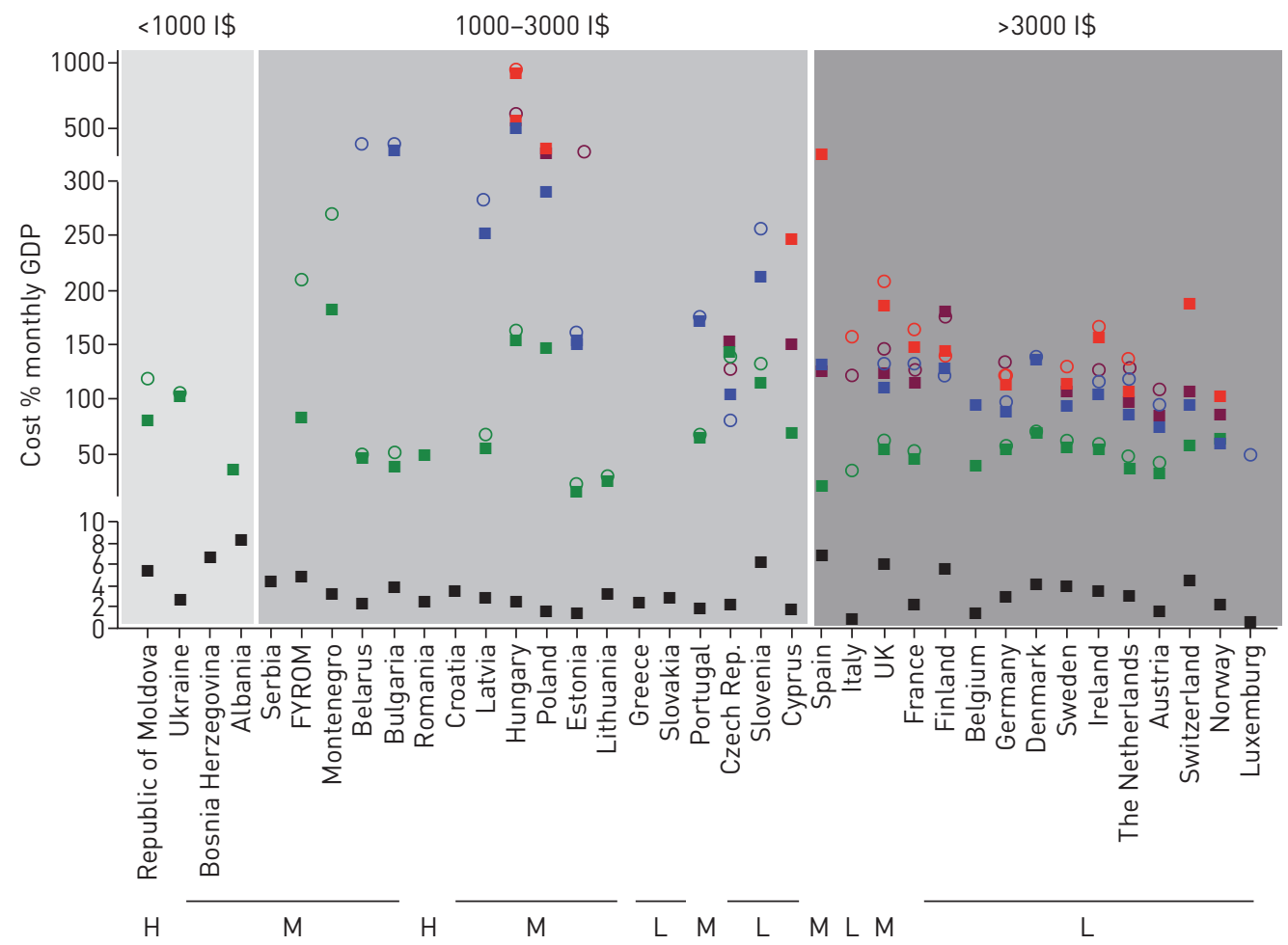

FIGURE 2 Affordability of treatment regimens. GDP: gross domestic product. Square: preferred regimen; circle: alternative regimen; black: pan-sensitive; green: multidrug resistant; red: extensively drug resistant (XDR); blue: pre-XDRinj TB (multidrug-resistant tuberculosis with additional resistance to second-line injectable); purple: pre-XDRfq TB (multidrugresistant tuberculosis with additional resistance to fluoroquninolone); $\mathrm{H}$ : high incidence (>100 per 100000 people); M: medium incidence (15-100 per 100000 people); L: low incidence ( $<15$ per 100000 people).

(pre-)XDR-TB are almost exclusively available in countries with a high GDP per capita ( $>3000 \mathrm{I} \$$ ). In some countries with a moderate GDP per capita (1000-3000 I\$), these regimens are available but represent a large part of a month's GDP. In the four countries with the lowest GDP per capita $(<1000$ I\$), only pan-sensitive and MDR-TB regimens are available.

\section{Discussion}

The results show that a standard first-line drug regimen against TB was available in each of the countries surveyed, but not adequate regimens for MDR-TB or (pre-)XDR-TB. The limited availability of second-line drugs corroborates those reported in a survey among five European MDR-TB treatment centres [13]. Costs and affordability differed markedly between countries, with drug costs for regimens for MDR-TB and (pre-)XDR-TB representing large parts of a monthly GDP per capita in a considerable number of countries, and not seldom exceeding $100 \%$. There was a clear trend of increasing drug cost with regimens against more extensive drug-resistant profiles.

The high drug cost associated with resistance against SL-inj and/or FQs is of concern given the increasing number of patients with (pre-)XDR-TB in European countries [14]. Reasons for the large differences observed in drug cost might be related to differences in procurement practices or accessibility to discounted prices through international donors or organisations. The recent past, in the field of HIV-treatment, has shown that concerted efforts from appropriate international bodies, politicians and patient-activists can influence availability and prices for live-saving drugs [15]. There is no reason why such efforts cannot be repeated for drugs needed to treat drug-resistant TB.

The Global Drug Facility (GDF), in collaboration with the Global Fund and other funding agencies, assists in the procurement of TB drugs for selected countries, either through grants or direct procurement [16]. This ensures a timely, affordable and quality-assured drug supply. Our analysis included nine countries that procured drugs with the GDF in 2011 (Republic of Moldova, Romania, Serbia, Belarus, Bulgaria, Estonia, Bosnia and Herzegovina, Ukraine, FYROM). Drug cost for a preferred MDR-TB regimen represented in four of these countries below 35\% of a month's GDP per capita while, in three other 
countries, the percentage was below $65 \%$, indicating the important role that the GDF plays by contributing to affordable TB control. However, none of these countries reported the availability of drugs needed to provide a preferred XDR-TB regimen, although Lzd and $\mathrm{Cfz}$ are available at GDF. Reasons for this observation remain unclear.

The study shows that kanamycin is markedly cheaper than other second-line injectable. Kanamycin was identified as the second-line injectable that had a larger effect on treatment success in the treatment of MDR-TB compared to capreomycin [17]. It is therefore surprising that only $24 \%$ of the countries reported the availability of this drug. In the same study, Pto/Eto showed a better effect compared with PAS. In our study, Pto/Eto was also less expensive and regimens including this drug represented less of a month's GDP than regimens including PAS.

Recent studies have shown that regimens containing Lzd for the treatment of XDR-TB result in more rapid culture conversion compared with regimens without Lzd $[18,19]$. The main drawback of Lzd is the high risk of adverse events, although lowering the dose from $600 \mathrm{mg}$ to $300 \mathrm{mg}$ might be beneficial [19]. It is therefore unfortunate that it is the most expensive group 5 drug and not available in every country.

The affordability indicator was provided as a reference point to how expensive a drug regimen is. The assessment of actual affordability for a healthcare system depends on a number of factors, including the actual number of drug-resistant patients in the country, the (TB-related) healthcare expenditure in relation to GDP, as well as the relevant, nationally chosen willingness-to-pay threshold. We did not attempt to make such an assessment of affordability or on the willingness-to-pay by national health systems, but aimed to describe estimates of drug cost for a single month a treatment.

Drug cost is one component of the total cost related to TB-control, but is frequently not reported separately in studies or health assessments. While it is acknowledged that human resources or hospitalization costs for TB-treatment can represent a substantial part of the total costs of treatment, our study shows that the cost for drugs can be very high and increases with more complex treatment regimens.

One potential limitation of our study is the data collection through a survey among single TB-reference centres in each country. The drug cost refers to those incurred by the treatment centres when obtaining these drugs from any source available to them, including procurement through national TB programmes (NTPs) or pharmacies. As drug prices are fixed in most countries, this cost is generalisable for the county. With TB-reference centres being an integral part of NTPs in most countries, the drug cost incurred by the centres reflects the prices for the NTPs. The survey approach precluded the report of prices by standardised volume of active ingredients, although formulation of the drugs in the different countries was comparable. Beside this, we were able to adhere to the international guidelines on reporting drug prices [20].

Our approach also precludes a clear distinction between brand products and the lowest-priced generic equivalent. Most likely, our data reflect a mix of these products. Yet, our estimates are within the same range as presented in earlier studies and reports [21]. Despite these limitations, the approach of using a well-established consortium with members strategically placed in TB care and control activities, enables us to report an overview of prices of single drugs and preferred and alternative TB treatment regimens.

Another limitation of our study is the absence of comprehensive information on the price of FDC, which can be lower than the addition of single drugs. The method to deal with FDC containing pyrazinamide and/or ethambutol in addition to isoniazid and rifampicin might have under- or over-estimated total dugs costs for all regimens. However, the relative price of pyrazinamide and ethambutol are so minimal in comparison with the other drugs combined in the non-pansensitive regimens, that we feel that the effect is negligible and that the approach is not jeopardising our conclusions.

Treatment of MDR-TB and XDR-TB in Europe is challenging due to fact that adequate regimens are not available in every country. Where these regimens are available, drug cost can be exorbitant and represent a substantial amount of GPD per capita. With the availability of new anti-TB drugs like bedaquiline and delamanid, prices for the treatment of patients with MDR-TB and XDR-TB will increase even further. For some patients, the current cost of those new drugs for a single day of therapy exceeds the cost of drugs for a complete course of standard 6-month treatment regimen for patients with pan-drug susceptible TB. There is a need for consorted efforts from pharmaceutical companies, politicians and patient-activists to make vital drugs for treatment of drug-resistant TB available and affordable in every single country. Preventing MDR-TB and XDR-TB in the first place is a priority for every TB-control programme.

\section{Acknowledgements}

The TBNET contributors (www.tb-net.org) were as follows: Claire Andrejak, Amiens, France; Despo Pieridou-Bagatzouni, Nicosia, Cyprus; Aase Bengard Anderson, Odense, Denmark; Olivera Bojovic, Niksic, Montenegro; Graham Bothamley, London, UK; Judith Bruchfeld, Stockholm, Sweden; Luigi R. Codecasa, Milan, Italy; Manfred Danilovits, Tartu, Estonia; Edita Davidaviciene, Vilnus, Lithuania; Paulina Dalemo, Stockholm, Sweden; Giorgos 
Dimopoulos, Athens, Greece; Raquel Duarte, Porto, Portugal; Hasan Hafizi, Tirana, Albania; Ildiko Horvath, Budapest, Hungary; Fusun Eyuboglu, Ankara, Turkey; Elmira Ibraim, Bucharest, Romania; Mateja Jankovic; Zagreb, Croatia; Boris Kan, Stockholm, Sweden; Emilia Kopecka, Prague, Czech Republic; Katarzyna Kruczak, Krakow, Poland; Galyna Kutsyna, Luhansk, Ukraine; Wiel de Lange, Groningen, the Netherlands; Vaira Leimane; Riga; Latvia; Ulrich Mack, Oslo, Norway; Juan Ruiz Manzano, Barcelona, Spain; Roumania Markova; Sofia; Bulgaria; Colm McDonald, Dublin, Ireland; Anne-Marie McLaughlin, Dublin, Ireland; Gjyle Mulliqi, Pristina, Kosovo; Inge Muylle, Brussels; Belgium; Dragica Pesut, Belgrade, Serbia; Veronika Polcova, Prague, Czech Republic; Rudolf Rumetshofer, Vienna, Austria; Doina Rusu, Chisinau, Moldova; Alena Skrahina, Minsk, Belarus; Nicolina Spiric, Banjaluca, Bosnia and Herzegovina; Ivan Solovic, Vysne Hagy, Slovakia; Petra Svetina-Sorli, Golnik, Slovenia; Martina Vasakova, Prague, Czech Republic; Tuula Vasankari, Helsinki, Finland; Piret Viiklepp, Tallinn, Estonia; Gil Wirz, Luxembourg, Luxembourg; Maja Zakoska, Skopje, FYROM; Jean-Pierre Zellweger, Bern, Switzerland.

\section{References}

1 Diel R, Vandeputte J, de Vries G, et al. Costs of tuberculosis disease in the European Union: a systematic analysis and cost calculation. Eur Respir J 2014; 43: 554-565.

2 Tanimura T, Jaramillo E, Weil D, et al. Financial burden for tuberculosis patients in low- and middle-income countries: a systematic review. Eur Respir J 2014; 43: 1763-1775.

3 Winston CA, Navin TR, Becerra JE, et al. Unexpected decline in tuberculosis cases coincident with economic recession - United States, 2009. BMC Public Health 2011; 11: 846.

4 Munro SA, Lewin SA, Smith HJ, et al. Patient adherence to tuberculosis treatment: a systematic review of qualitative research. PLoS Med 2007; 4: e238.

5 Arinaminpathy N, Dye C. Health in financial crises: economic recession and tuberculosis in Central and Eastern Europe. J R Soc Interface 2010; 7: 1559-1569.

6 Karanikolos M, Mladovsky P, Cylus J, et al. Financial crisis, austerity, and health in Europe. Lancet 2013; 381 : 1323-1331.

7 Giehl C, Lange C, Duarte R, et al. TBNET - Collaborative research on tuberculosis in Europe. Eur J Microbiol Immunol 2012; 2: 264-274.

8 Guidelines for the treatment of tuberculosis. 4th ed. Geneva, World Health Organization, 2010.

9 Guidelines for the programmatic management of drug-resistant tuberculosis: 2011 update. Geneva, World Health Organization, 2011.

10 Lange C, Abubakar I, Alffenaar JW, et al. Management of patients with multidrug-resistant/extensively drug-resistant tuberculosis in Europe: a TBNET consensus statement. Eur Respir J 2014; 44: 23-63.

11 World Bank. PPP. conversion factor, GDP (LCU per international \$). http://data.worldbank.org/indicator/PA.NUS. PPP Date last accessed: April 192014

12 World Bank. GDP. per capita, PPP (current international \$). http://data.worldbank.org/indicator/NY.GDP.PCAP. PP.CD Date last accessed: April 192014

13 Sotgiu G, D'Ambrosio L, Centis R, et al. Availability of anti-tuberculosis drugs in Europe. Eur Respir J 2012; 40: 500-503.

14 Van derWerf M, Kodmon C, Hollo V, et al. Drug resistance among tuberculosis cases in the European Union and European Economic Area, 2007 to 2012. Euro Surveill 2014:19; pii=20733.

15 Hoen E’t, Berger J, Calmy A, et al. Driving a decade of change: HIV/AIDS, patents and access to medicines for all. J Int AIDS Soc 2011; 14: 15.

16 Arinaminpathy N, Cordier-Lassalle T, Vijay A, et al. The Global Drug Facility and its role in the market for tuberculosis drugs. Lancet 2013; 382: 1373-1379.

17 Ahuja SD, Ashkin D, Avendano M, et al. Multidrug resistant pulmonary tuberculosis treatment regimens and patient outcomes: an individual patient data meta-analysis of 9,153 Patients. PLoS Med 2012; 9: e1001300.

18 Sotgiu G, Centis R, D'Ambrosio L, et al. Efficacy, safety and tolerability of linezolid containing regimens in treating MDR-TB and XDR-TB: systematic review and meta-analysis. Eur Respir J 2012; 40: 1430-1442.

19 Lee M, Lee J, Carroll MW, et al. Linezolid for treatment of chronic extensively drug-resistant tuberculosis. $N$ Engl J Med 2012; 367: 1508-1518.

20 Shi L, Hodges M, Drummond M, et al. Good research practices for measuring drug costs in cost-effectiveness analyses: an international perspective: the ISPOR Drug Cost Task Force report-Part VI. Value Health 2010; 13: $28-33$.

21 DR-TB drugs Under the Microscope: The Sources and Prices of Medicines for Drug-Resistant Tuberculosis. 3rd ed. Paris, MSF/IUATLD, 2013. 